\title{
Noncommutative geometry inspired 3-dimensional charged black hole solution in an anti-de Sitter background spacetime
}

\author{
Farook Rahaman $^{1, \mathrm{a}}$, Piyali Bhar ${ }^{1, \mathrm{~b}}$, Ranjan Sharma ${ }^{2, \mathrm{c}}$, Rishi Kumar Tiwari ${ }^{3, \mathrm{~d}}$ \\ ${ }^{1}$ Department of Mathematics, Jadavpur University, Kolkata 700032, West Bengal, India \\ 2 Department of Physics, P. D. Women's College, Jalpaiguri 735101, India \\ ${ }^{3}$ Department of Mathematics, Govt. Model Science College, Rewa, MP, India
}

Received: 22 November 2014 / Accepted: 15 February 2015 / Published online: 5 March 2015

(C) The Author(s) 2015. This article is published with open access at Springerlink.com

\begin{abstract}
We report a 3-D charged black hole solution in an anti-de Sitter space inspired by noncommutative geometry. In this construction, the black hole exhibits two horizons, which turn into a single horizon in the extreme case. We investigate the impacts of electromagnetic field on the location of the event horizon, mass and thermodynamic properties such as Hawking temperature, entropy, and heat capacity of the black hole. The geodesics of the charged black hole are also analyzed.
\end{abstract}

\section{Introduction}

In recent years, noncommutative geometry has received a considerable interest to get insight into many conceptual issues in the quantum domain of high energy physics. It is argued that at sufficiently high energy scales the targetspace structure coordinates may become noncommuting operators on a D-brane [1,2]. Consequently, the noncommutativity of the spacetime can be encoded in a commutator $\left[x^{\mu}, x^{\nu}\right]=i \theta^{\mu \nu}$, where $\theta^{\mu \nu}$ is an antisymmetric matrix of dimension [length $]^{2}$ which determines the fundamental cell discretization of the spacetime. It is similar to the way the Planck constant $\hbar$ discretizes phase space [3]. The noncommutative approach provides the construction of a black hole with a minimum scale $\sqrt{\theta}$, known as noncommutative black hole $[4,5]$, whose commutative limit is the Schwarzschild metric. It is noteworthy that noncommutative geometry is an intrinsic property

\footnotetext{
a e-mail: farook_rahaman@yahoo.com; rahaman@iucaa.ernet.in

be-mail: piyalibhar90@gmail.com

ce-mail: rsharma@iucaa.ernet.in

d e-mail: rishitiwari59@rediffmail.com
}

of the spacetime and does not depend on the curvature of spacetime.

A large number of studies inspired by noncommutative geometry are available in the literature. Myung et al. [6] have discussed thermodynamic behavior and evaporation processes involved in a noncommutative black hole. In their investigations, they have considered a noncommutative 3dimensional black hole whose commutative limit is the nonrotating Bañados et al. [7] (henceforth BTZ) black hole. Nozari and Mehdipour [8] have investigated the Hawking radiation from a noncommutative black hole. Rahaman et al. [9], in the context of galactic rotation curves, have shown that a noncommutative geometrical background is sufficient for the existence of a stable circular orbit and one need not consider dark matter in this formalism. In a separate paper, Rahaman et al. [10] have shown that wormhole solutions exist up to 5-dimensional spacetimes; however, they do not exist in dimensions $>5$. Banerjee et al. [11] have investigated the thermodynamic properties (Hawking temperature, entropy, and area law, in particular) of a Schwarzschild black hole in noncommutative spacetimes. By invoking a graphical analysis, the authors have shown the non-trivial behavior of the noncommutative Schwarzschild metric at different length scales. It has been shown that in the limit of large $r(r \gg \sqrt{\theta})$ outside the horizon, the metric behaves like the standard Schwarzschild solution, whereas in the limit of small $r(r \ll \sqrt{\theta})$ inside the horizon, the spacetime is de Sitter type with a constant positive curvature.

The motivation of the present work is to construct a 3-dimensional charged black hole solution based on noncommutative geometry in an anti-de Sitter background spacetime. Using the commutator of operators corresponding to spacetime coordinates, i.e. (the integer D below is even), one can encode the noncommutativity of spacetime $\left[x^{\mu}, x^{\nu}\right]=$ $i \theta^{\mu \nu}$. Here the antisymmetric matrix $\theta^{\mu \nu}$ assumes the blockdiagonal form as $\theta^{\mu \nu}=\operatorname{diag}\left(\theta_{1}, \ldots, \theta_{D / 2}\right)$ where 
$\theta_{i}=\left[\begin{array}{cc}0 & 1 \\ -1 & 0\end{array}\right]$ for all, $i=1,2, \ldots, D / 2$

The fundamental discretization of spacetime can be determined by this antisymmetric matrix $\theta^{\mu \nu}$. For flat spacetimes, it is also recognized that noncommutativity removes the point-like structures in favor of smeared objects. The fundamental discretization of spacetime can be determined by this antisymmetric matrix $\theta^{\mu \nu}$. For flat spacetimes, it is also recognized that noncommutativity removes the pointlike structures in favor of smeared objects. One can mathematically implement the effect of the smearing with a substitution of the Dirac-delta function by a Gaussian distribution of minimal length $\sqrt{\theta}$. In particular, here we will consider the energy density of a static and spherically symmetric, smeared and particle-like gravitational source. Since we assumed this particular form of the energy density $\rho$ of the fluid (which is smeared and particle-like gravitational source and will be given in Eq. (15) in the next section), therefore, we call the solutions 'noncommutative geometry inspired'.

It is to be stressed here that gravitational analyses in $(2+1)$-dimensions have become extremely fascinating with the discovery of the black hole solution by Bañados et al. [7]. The BTZ metric describes the exterior gravitational field of a circularly symmetric Einstein-Maxwell system in the presence of a negative cosmological constant and is characterized by its mass, angular momentum, and charge. In standard $(3+1)$-dimensions, Einstein-Maxwell systems for static spherically symmetric charged distributions of matter have investigated by many (see for example, Tikekar and Singh [12], Sharma et al. [13], Thirukkanesh and Maharaj [14] and also references therein). Analytic models of static charged fluid spheres has been reviewed by Ivanov [15]. In $(2+1)$-dimensions, Rahaman et al. [16] have developed a model for a charged gravastar in an anti-de Sitter background spacetime. Corresponding to the static BTZ exterior, Cataldo et al. [17] have investigated the properties of a circularly symmetric static charged fluid distribution. Larrañaga and Tejeiro [18] have obtained a charged black hole solution in 3-D anti-de Sitter space inspired by noncommutative geometry where a Gaussian distribution of charge has been considered. In an earlier work, Rahaman et al. [19] have studied BTZ black holes inspired by noncommutative geometry. The current investigation is an extension of Ref. [19] where we have incorporated a distribution of charge into the system. The resultant charged black hole appears to have two horizons, which, however, get reduced to a single horizon in the extreme case. Thermodynamic properties such as Hawking temperature, entropy and heat capacity of the charged black hole have been analyzed. Effective potential and behavior of test particles around the black hole have also been outlined.

\section{Interior spacetime}

We write the line element for the interior spacetime of a static circularly symmetric charged distribution of matter in $(2+1)$-dimensions in the form

$\mathrm{d} s^{2}=-e^{\nu(r)} \mathrm{d} t^{2}+e^{\lambda(r)} \mathrm{d} r^{2}+r^{2} \mathrm{~d} \phi^{2}$.

The energy-momentum tensor corresponding to the charged fluid distribution is assumed to be

$$
\begin{aligned}
T_{i j}^{\text {Total }}= & \left(\rho+p_{r}\right) u_{i} u_{j}-p_{r} g_{i j}+\left(p_{t}-p_{r}\right) \eta_{i} \eta_{j} \\
& -\frac{1}{4 \pi}\left(F_{a}^{c} F_{b c}-\frac{1}{4} g_{a b} F_{c d} F^{c d}\right),
\end{aligned}
$$

where $\rho$ is the energy density, $p_{r}$ is the radial pressure and $p_{t}$ is the tangential pressure. In (2), $u^{i}$ is the velocity of the fluid and $\eta^{i}$ is a radial unit vector satisfying the relation $u^{i} u_{i}=$ $-\eta^{i} \eta_{j}=1$. The electromagnetic field tensor is governed by the Maxwell equations

$F_{; j}^{i j}=-4 \pi J^{i}$

where $J^{\mu}$ is the current 3-vector defined as

$J^{i}=\sigma(r) u^{i}$.

In (4), $\sigma(r)$ is the proper charged density of the distribution. The electromagnetic field tensor may be expressed as

$F_{i j}=E(r)\left(\delta_{i}^{t} \delta_{j}^{r}-\delta_{i}^{r} \delta_{j}^{t}\right)$,

where $E(r)$ is the electric field intensity. In the presence of a negative cosmological constant $(\Lambda<0)$, the EinsteinMaxwell equations,

$R_{i j}-\frac{1}{2} R g_{i j}+\Lambda g_{i j}=-2 \pi T_{i j}^{\text {Total }}$,

for the metric (1) yield the following set of four independent equations (in geometrized units $G=c=1$ ):

$\frac{\lambda^{\prime} e^{-\lambda}}{2 r}=2 \pi \rho+E^{2}+\Lambda$,

$\frac{v^{\prime} e^{-\lambda}}{2 r}=2 \pi p_{r}-E^{2}-\Lambda$,

$\frac{e^{-\lambda}}{2}\left(\frac{\nu^{\prime 2}}{2}+v^{\prime \prime}-\frac{1}{2} \nu^{\prime} \lambda^{\prime}\right)=2 \pi p_{t}+E^{2}-\Lambda$,

$\sigma(r)=\frac{e^{-\frac{\lambda}{2}}}{4 \pi r}(r E)^{\prime}$,

where a prime denotes differentiation with respect to the radial parameter $r$. Equation (10) can equivalently be expressed as

$E(r)=\frac{4 \pi}{r} \int_{0}^{r} \tilde{r} \sigma(\tilde{r}) e^{\frac{\lambda(\tilde{r})}{2}} \mathrm{~d} \tilde{r}=\frac{q(r)}{r}$,

where $q(r)$ is the total charge contained within a sphere of radius $r$. 
We assume that the volume charge density has the form

$\sigma(r) e^{\frac{\lambda(r)}{2}}=\sigma_{0} r^{n}$,

where $n$ is an arbitrary constant and $\sigma_{0}$ is the central charged density. Though it is true that one is free to choose an infinite number of possible ansatz, the main motivation for a particular choice in our model was to generate solutions which could be considered as physically meaningful. Secondly, solutions obtained earlier for the neutral case can easily be regained from our solution for the specified ansatz. This enables one to investigate the effects of electromagnetic field on the system explicitly. The choice (12) of the charge density is only dictated by the mathematical convenience and has no physical justification since it gives the energy divergent at infinity. However, this particular choice is different from the Gaussian distribution of charge assumed in Ref. [18]. We will see that solutions obtained earlier for the neutral case can easily be regained from our solution for the specified ansatz.

Consequently, from Eq. (11) we get

$E(r)=\frac{4 \pi \sigma_{0}}{n+2} r^{n+1}$,

$q(r)=\frac{4 \pi \sigma_{0}}{n+2} r^{n+2}$.

Further, we consider the maximally localized source of energy of the static spherically symmetric charge distribution as a Gaussian distribution with minimal width $\sqrt{\theta}$ expressed as [19],

$\rho=\frac{M}{4 \pi \theta} e^{-\frac{r^{2}}{4 \theta}}$,

where $M$ is the total mass of the source diffused throughout a region of linear dimension $\sqrt{\theta}$.

Substituting (13) and (15) in (7), we obtain

$\frac{\lambda^{\prime} e^{-\lambda}}{2 r}=\frac{M}{2 \theta} e^{-\frac{r^{2}}{4 \theta}}+\left(\frac{4 \pi \sigma_{0}}{n+2}\right)^{2} r^{2 n+2}+\Lambda$,

which, on integration, yields

$e^{-\lambda}=-A+2 M e^{-\frac{r^{2}}{4 \theta}}-\frac{1}{n+2}\left(\frac{4 \pi \sigma_{0}}{n+2}\right)^{2} r^{2 n+4}-\Lambda r^{2}$,

where $A$ is a constant of integration. In Ref. [9] it has been shown that the constant of integration $A$ plays the role of the mass of the black hole, i.e., $A=M$. In our construction, if we set $\sigma_{0}=0$ and consider the limit $\frac{r}{\sqrt{\theta}} \rightarrow \infty$, then it follows that $A=M$. Accordingly, we rewrite Eq. (17) as

$e^{-\lambda}=-M+2 M e^{-\frac{r^{2}}{4 \theta}}-\frac{1}{n+2}\left(\frac{4 \pi \sigma_{0}}{n+2}\right)^{2} r^{2 n+4}-\Lambda r^{2}$.

Note that the vacuum Einstein field equations in $(2+1)$ dimensional spacetime in the presence of a negative cos- mological constant $(\Lambda<0)$ admit a black hole solution known as BTZ [7] solution for which we have $g_{r r}=\left(g_{t t}\right)^{-1}$. To retain the structure of BTZ black hole, we must have $e^{v}=e^{-\lambda}$. From Eqs. (7)-(8), it then follows that

$p_{r}=-\rho$,

which is the ' $\rho$ vacuum' EOS in reference to 'zero-point energy of quantum fluctuations' [19].

Combining Eqs. (7)-(9) and (19), we obtain

$\frac{e^{-\lambda}}{2}\left(\lambda^{\prime 2}-\lambda^{\prime \prime}\right)=2 \pi p_{t}+E^{2}-\Lambda$.

From Eq. (16), we get

$$
\begin{aligned}
e^{-\lambda}\left(\lambda^{\prime \prime}-\lambda^{\prime 2}\right)= & \frac{M}{\theta} e^{-\frac{r^{2}}{4 \theta}}\left(1-\frac{r^{2}}{2 \theta}\right) \\
& +2(2 n+3)\left(\frac{4 \pi \sigma_{0}}{n+2}\right)^{2} r^{2 n+2}+2 \Lambda .
\end{aligned}
$$

Combining Eqs. (20) and (21), we obtain

$p_{t}=-\frac{M}{\pi \theta} e^{-\frac{r^{2}}{4 \theta}}\left(1-\frac{r^{2}}{2 \theta}\right)-\frac{n+2}{\pi}\left(\frac{4 \pi \sigma_{0}}{n+2}\right)^{2} r^{2 n+2}$.

We, thus, have a noncommutative geometry inspired analytic model of a 3-D charged black hole. In this construction, the spacetime near the origin behaves as

$$
\begin{aligned}
e^{v}=e^{-\lambda}= & -M+2 M\left[1-\frac{r^{2}}{4 \theta}+\frac{r^{4}}{32 \theta^{2}}+\mathcal{O}\left(r^{8}\right)\right] \\
& -\frac{1}{n+2}\left(\frac{4 \pi \sigma_{0}}{n+2}\right)^{2} r^{2 n+4}-\Lambda r^{2} .
\end{aligned}
$$

Note that, for $n=0$, by adjusting the parameters suitably, one can regain the spacetime for the $(2+1)$-dimensional static charged distribution

$e^{\nu}=e^{-\lambda}=M-\left[\frac{M}{2 \theta}+\Lambda\right] r^{2}+\left[\frac{2 M}{32 \theta^{2}}-2 \pi^{2} \sigma_{0}^{2}\right] r^{4}$,

obtained earlier by Liang and Liu [20].

In the following sections, we shall analyze some features of our charged BTZ black hole.

\section{Features of the charged BTZ black hole}

\subsection{Formation of event horizons}

The condition for the formation of event horizon is given by $g_{t t}\left(r_{h}\right)=0$, which implies 


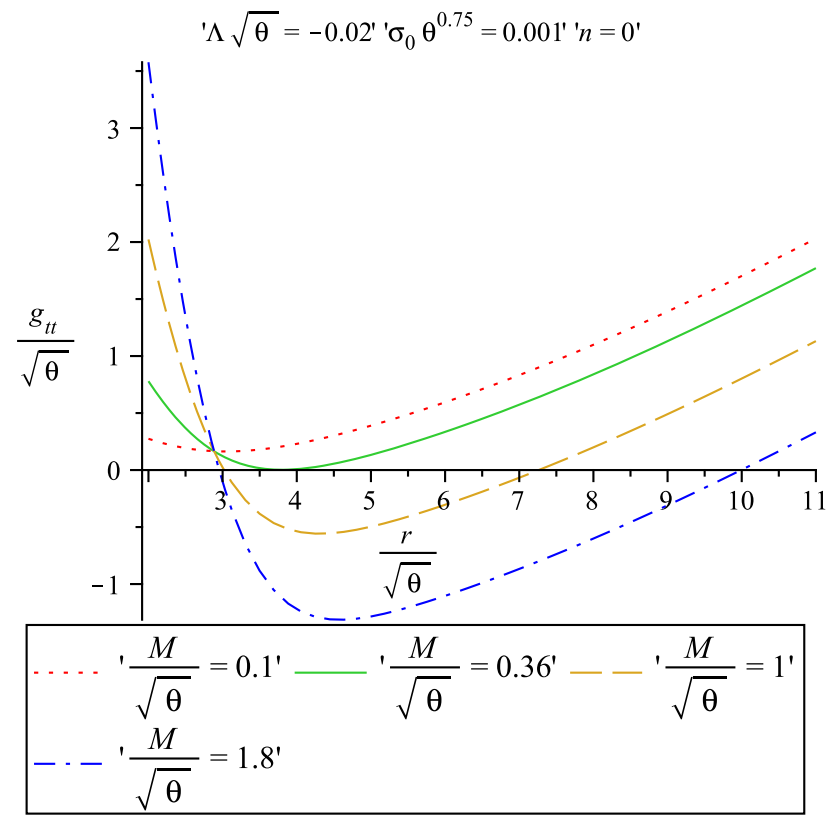

Fig. $1 \frac{g_{t t}}{\sqrt{\theta}}$ plotted against $\frac{r}{\sqrt{\theta}}$. We have assumed $M=0.1 \sqrt{\theta}$ (dotted curve), $M=0.36 \sqrt{\theta}$ (solid curve), $M=1 \sqrt{\theta}$ (dashed curve), and $M=1.8 \sqrt{\theta}$ (dot-dashed curve)

$-M+2 M e^{-\frac{r_{h}^{2}}{4 \theta}}-\frac{1}{n+2}\left(\frac{4 \pi \sigma_{0}}{n+2}\right)^{2} r_{h}^{2 n+4}-\Lambda r_{h}^{2}=0$,

where $r_{h}$ is the horizon distance. Though a closed-form solution for $r_{h}$ cannot be obtained from the above equation, one can express the mass parameter in terms of $r_{h}$ as

$M=\frac{\frac{1}{n+2}\left(\frac{4 \pi \sigma_{0}}{n+2}\right)^{2} r_{h}^{2 n+4}+\Lambda r_{h}^{2}}{-1+2 e^{-\frac{r_{h}^{2}}{4 \theta}}}$

In Fig. 1, we show the existence of horizons and their corresponding radii by identifying the intersections of $\frac{g_{t t}}{\sqrt{\theta}}$ with $\frac{r}{\sqrt{\theta}}$. We note that, for a given value of $\Lambda \sqrt{\theta}=-0.02$, there exists a minimal mass $M_{0}=0.36 \sqrt{\theta}$ below which no black hole exists though two distinct horizons exist for $M>0.36 \sqrt{\theta}$. If mass of the black hole increases, the distance between the two horizons increases. The two horizons coincide at a minimal mass $M=M_{0}$. In our illustrative example, $r=r_{0}=3.7 \sqrt{\theta}$ is the distance where the two horizons coincide, i.e., $r_{0}$ is the radius of the extremal black hole. The results may be summarized as follows:

- Existence of two horizons for $\frac{M}{\sqrt{\theta}}>M_{0}$.

- One horizon corresponding to the extremal black hole with $\frac{M}{\sqrt{\theta}}=M_{0}$.

- No horizon for $\frac{M}{\sqrt{\theta}}<M_{0}$.

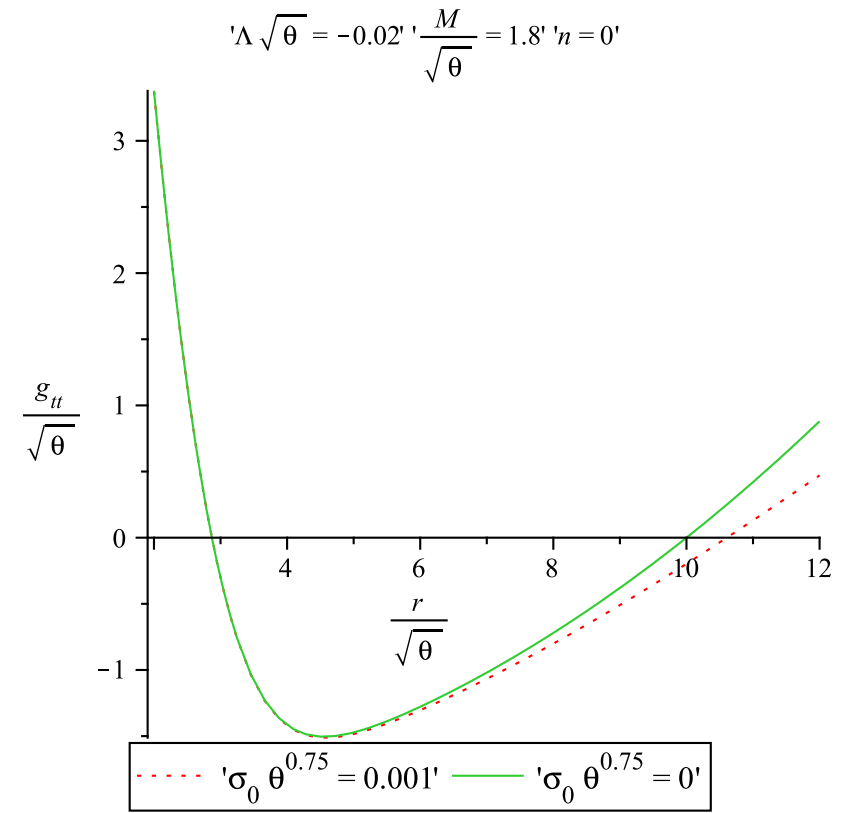

Fig. $2 \frac{g_{t t}}{\sqrt{\theta}}$ plotted against $\frac{r}{\sqrt{\theta}}$ for two different cases: (1) $\sigma_{0} \theta^{.75}=$ 0.001 (dotted curve) and (2) $\sigma_{0}=0$ (solid curve)

From Eq. (26) it is interesting to note that the lower limit mass $M_{0}$ increases when charge is incorporated into the system. Moreover, the distance of the outer event horizon increases in the presence of electromagnetic field as shown in Fig. 2.

In Figs. 3 and 4, we show the behavior of the density parameter $\rho \sqrt{\theta}$ : radial pressure $\left(p_{r} \sqrt{\theta}\right)$ and tangential pressure $\left(p_{t} \sqrt{\theta}\right)$, respectively, between the inner and outer horizons and outside the outer horizon for different choices of the mass parameter. Variation of mass $\frac{M}{\sqrt{\theta}}$ with respect to the horizon radius $\frac{r_{h}}{\sqrt{\theta}}$ is shown in Fig. 5. We note a decrease in mass $\frac{M}{\sqrt{\theta}}$ within the horizon distance $\frac{r_{h}}{\sqrt{\theta}}$ when charge is incorporated into system. This is shown in Fig. 6.

\subsection{Thermodynamical properties}

The Hawking temperature,

$T_{H}=\left.\frac{1}{4 \pi}\left(\frac{\mathrm{d} g_{t t}}{\mathrm{~d} r}\right) \sqrt{-g^{t t} g^{r r}}\right|_{r=r_{h}}$,

in our model turns out to be

$T_{H}=-\frac{r_{h}}{2 \pi}\left[\frac{M}{2 \theta} e^{-\frac{r_{h}^{2}}{4 \theta}}+r_{h}^{2 n+2}\left(\frac{4 \pi \sigma_{0}}{n+2}\right)^{2}+\Lambda\right]$.

The surface gravity,

$\kappa=\left.\frac{1}{2}\left(\frac{\mathrm{d} g_{t t}}{\mathrm{~d} r}\right)\right|_{r=r_{h}}$, 


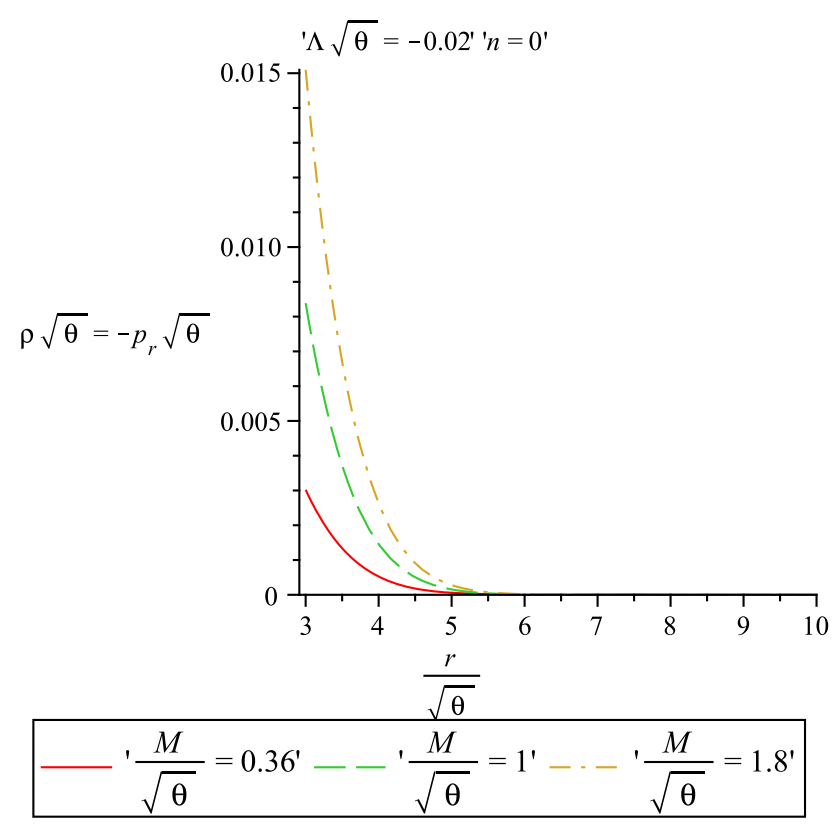

Fig. $3 \rho \sqrt{\theta}$ plotted against $\frac{r}{\sqrt{\theta}}$. We have assumed $M=0.36 \sqrt{\theta}$ (solid curve), $M=1 \sqrt{\theta}$ (dashed curve), and $M=1.8 \sqrt{\theta}$ (dot-dashed curve)

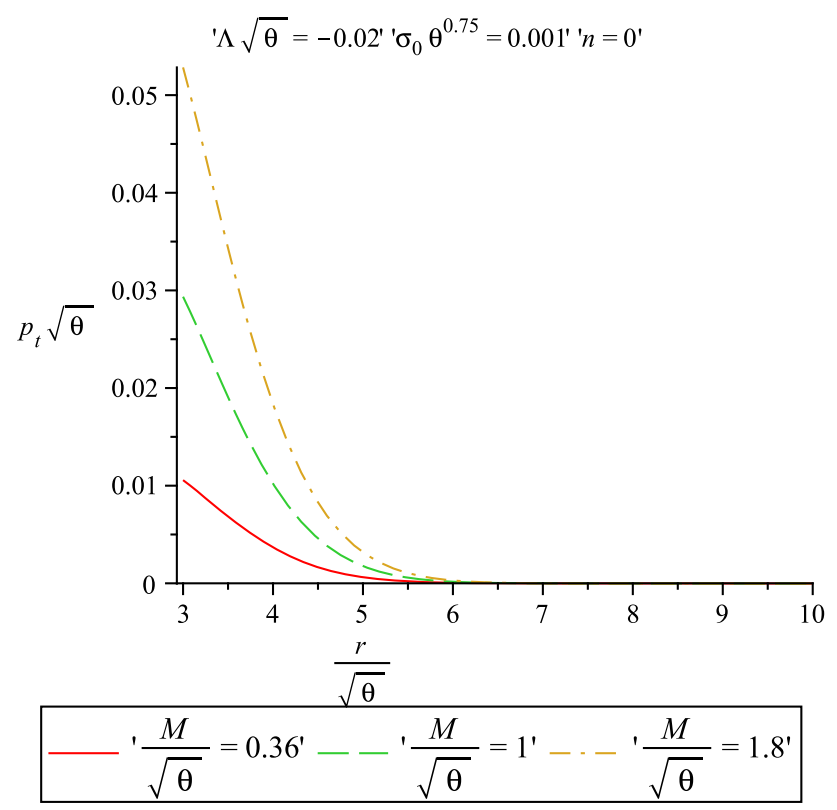

Fig. $4 p_{t} \sqrt{\theta}$ plotted against $\frac{r}{\sqrt{\theta}}$. We have assumed $M=0.36 \sqrt{\theta}$ (solid curve), $M=1 \sqrt{\theta}$ (dashed curve), and $M=1.8 \sqrt{\theta}$ (dot-dashed curve)

takes the form

$\kappa=-r_{h}\left[\frac{M}{2 \theta} e^{-\frac{r_{h}^{2}}{4 \theta}}+r_{h}^{2 n+2}\left(\frac{4 \pi \sigma_{0}}{n+2}\right)^{2}+\Lambda\right]$,

and the Bekenstein-Hawking entropy can be obtained from the equation

$S=4 \pi r_{h}$.

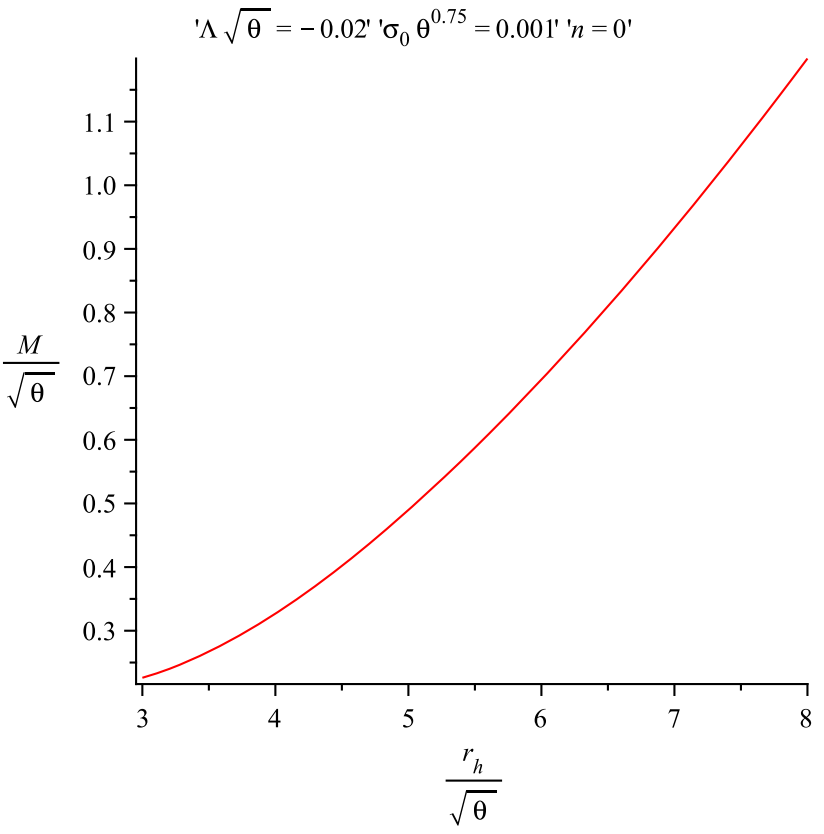

Fig. 5 Variation of mass $\frac{M}{\sqrt{\theta}}$ with respect to the horizon radius $\frac{r_{h}}{\sqrt{\theta}}$

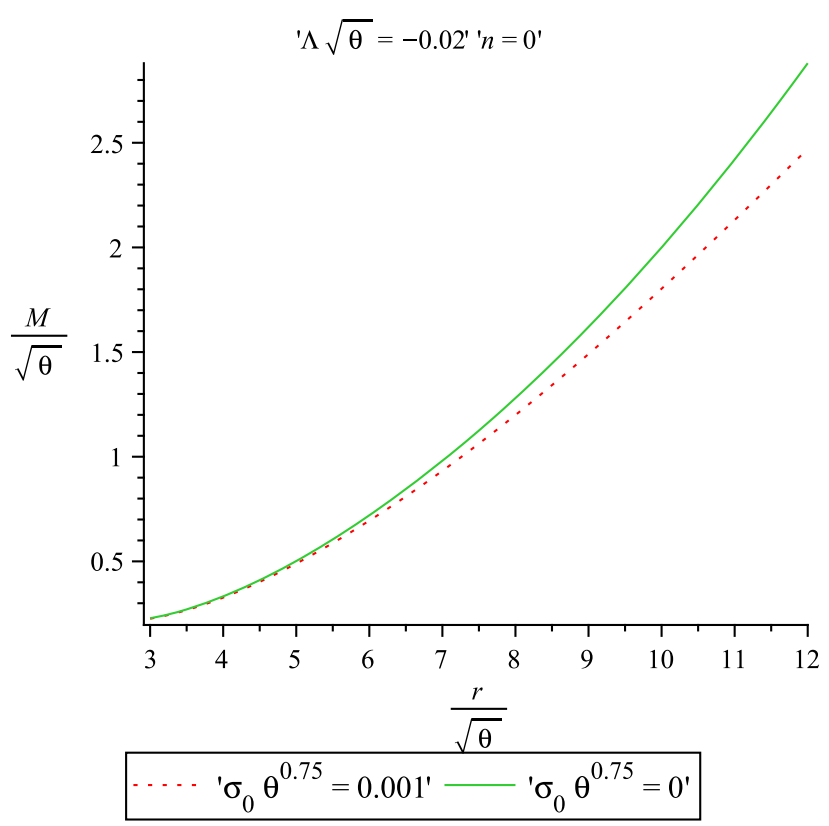

Fig. 6 Variation of mass $\frac{M}{\sqrt{\theta}}$ with respect to the horizon radius $\frac{r_{h}}{\sqrt{\theta}}$ for (1) $\sigma_{0} \theta^{.75}=0.001$ (dotted curve) and (2) $\sigma_{0}=0$ (solid curve)

In Fig. 7, we show the variation of the Hawking temperature $T_{H}$ with respect to the radial distance $\frac{r}{\sqrt{\theta}}$. Interestingly, the Hawking temperature $T_{H}$ decreases with the inclusion of the charge as shown in Fig. 8.

The heat capacity of the charged BTZ black hole can be obtained from the relation

$C=\frac{\partial M\left(r_{h}\right)}{\partial T\left(r_{h}\right)}=\frac{\partial M\left(r_{h}\right)}{\partial r_{h}} \frac{1}{\frac{\partial T\left(r_{h}\right)}{\partial r_{h}}}$. 


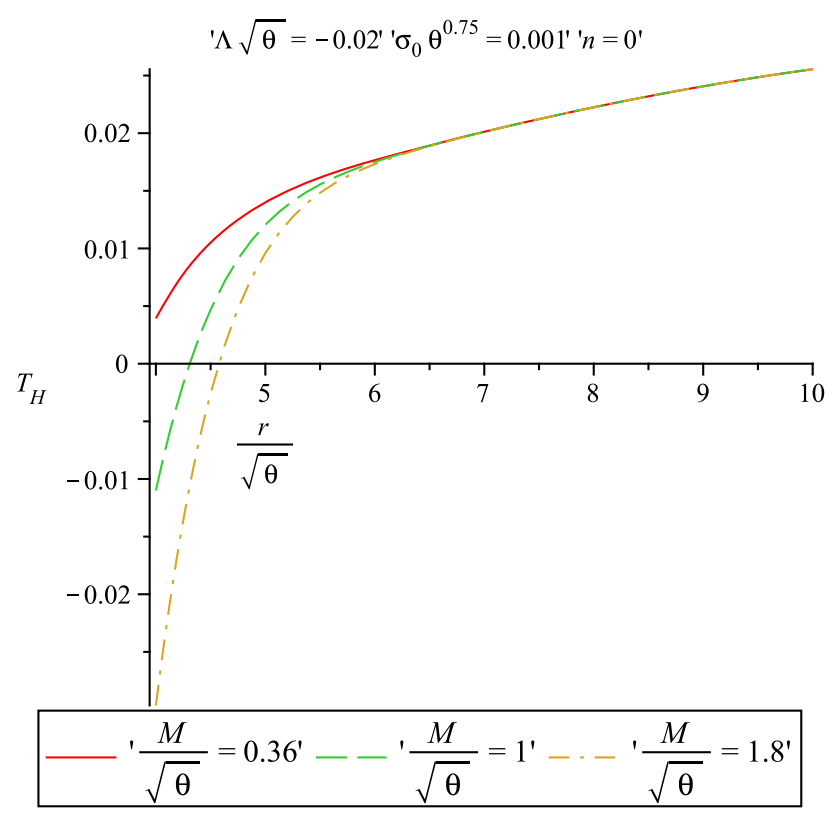

Fig. 7 Hawking temperature $T_{H}$ plotted against $\frac{r}{\sqrt{\theta}}$. We have assumed $M=0.36 \sqrt{\theta}$ (solid curve), $M=1 \sqrt{\theta}$ (dashed curve), and $M=$ $1.8 \sqrt{\theta}$ (dot-dashed curve)

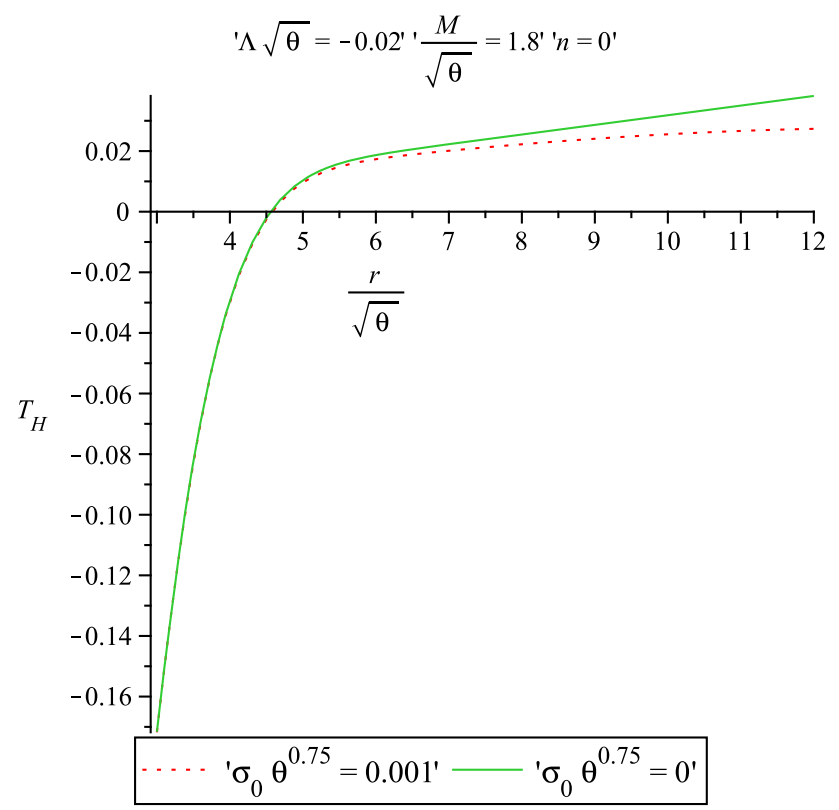

Fig. 8 The Hawking temperature $T_{H}$ for (1) $\sigma_{0} \theta^{.75}=0.001$ (dotted curve) and (2) $\sigma_{0}=0$ (solid curve)

For stability of the black hole, $C$ has to be positive [20]. In Fig. 9, the nature of the heat capacity in our model is shown. We note that $C$ vanishes at the extremal event horizon $r_{0}$. Since $C$ is negative in the region $\frac{r_{h}}{\sqrt{\theta}}<\frac{r_{0}}{\sqrt{\theta}}$, it is of no physical interest. However, for $\frac{r_{h}}{\sqrt{\theta}}>\frac{r_{0}}{\sqrt{\theta}}, C$ is positive, which indicates that the noncommutative geometry inspired charged BTZ black hole is stable in this region. We notice an

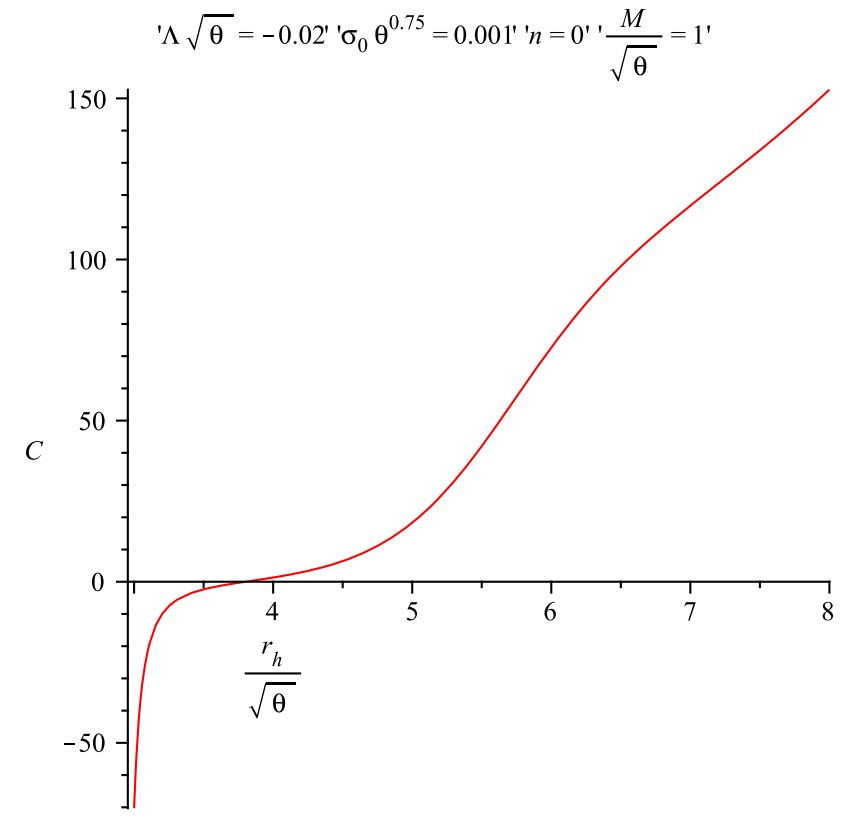

Fig. 9 Heat capacity $C$ plotted against $\frac{r_{h}}{\sqrt{\theta}}$

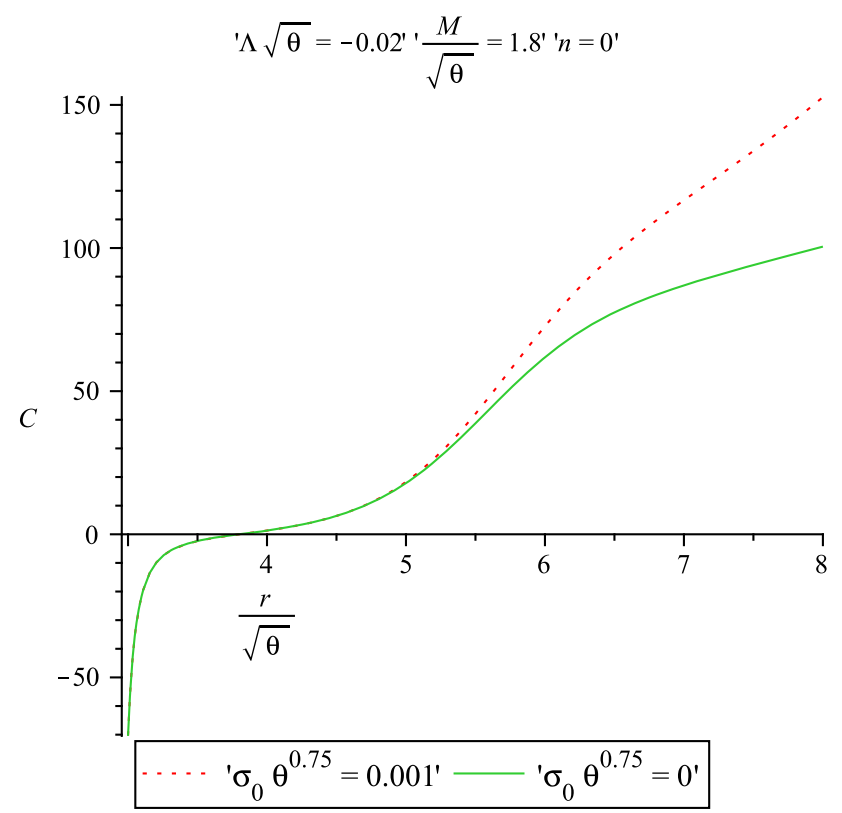

Fig. 10 Heat capacity $C$ for (1) $\sigma_{0} \theta^{.75}=0.001$ (dotted curve) and (2) $\sigma_{0}=0$ (solid curve $)$

appreciable change in the heat capacity due to the inclusion of charge as shown in Fig. 10.

\subsection{The geodesics}

The geodesics equation,

$\frac{\mathrm{d} x^{\mu}}{\mathrm{d} \tau^{2}}+\Gamma_{\nu \lambda}^{v} \frac{\mathrm{d} x^{\nu}}{\mathrm{d} \tau} \frac{\mathrm{d} x^{\lambda}}{\mathrm{d} \tau}=0$ 
in our construction yields the following results:

$$
\begin{aligned}
\frac{1}{f(r)}\left(\frac{\mathrm{d} r}{\mathrm{~d} \tau}\right)^{2} & =\frac{\Sigma^{2}}{f(r)}-\frac{p^{2}}{r^{2}}+L, \\
r^{2}\left(\frac{\mathrm{d} \phi}{\mathrm{d} \tau}\right) & =p, \\
\frac{\mathrm{d} t}{\mathrm{~d} \tau} & =\frac{\Sigma}{f(r)},
\end{aligned}
$$

where

$f(r)=-M+2 M e^{-\frac{r^{2}}{4 \theta}}-\frac{1}{n+2}\left(\frac{4 \pi \sigma_{0}}{n+2}\right)^{2} r^{2 n+4}-\Lambda r^{2}$

The constants $\Sigma$ and $p$ represent the energy per unit mass and angular momentum, respectively. In Eqs. (33)-(35), $\tau$ is the affine parameter and $L$ is the Lagrangian having values 0 and -1 for massless and massive particles, respectively. From Eq. (33), we have

$\frac{1}{2}\left(\frac{\mathrm{d} r}{\mathrm{~d} \tau}\right)^{2}=\frac{1}{2}\left[\Sigma^{2}+f(r)\left(L-\frac{p^{2}}{r^{2}}\right)\right]$.

Combining Eqs. (35) and (36), we get

$$
\left(\frac{\mathrm{d} r}{\mathrm{~d} t}\right)^{2}=\{f(r)\}^{2}\left[1+\frac{f(r)}{\Sigma^{2}}\left(L-\frac{p^{2}}{r^{2}}\right)\right] .
$$

Comparing Eq. (36) with

$\frac{1}{2}\left(\frac{\mathrm{d} r}{\mathrm{~d} \tau}\right)^{2}+V_{\mathrm{eff}}=0$

we obtain the effective potential in the form

$V_{\mathrm{eff}}=-\frac{1}{2}\left[\Sigma^{2}+f(r)\left(L-\frac{p^{2}}{r^{2}}\right)\right]$.

\subsubsection{Null geodesics}

For a massless particle, i.e., photon with $L=0$ and $p=0$, we have

$$
\begin{aligned}
& \left(\frac{\mathrm{d} r}{\mathrm{~d} t}\right)^{2} \\
& =\left[-M+2 M e^{-\frac{r^{2}}{4 \theta}}-\frac{1}{n+2}\left(\frac{4 \pi \sigma_{0}}{n+2}\right)^{2} r^{2 n+4}-\Lambda r^{2}\right]^{2},
\end{aligned}
$$

which in integral form can be expressed as

$$
\pm t=\int_{\frac{r_{h}}{\sqrt{\theta}}}^{\frac{r^{*}}{\sqrt{\theta}}} \frac{\mathrm{d} r}{-M+2 M e^{-\frac{r^{2}}{4 \theta}}-\frac{1}{n+2}\left(\frac{4 \pi \sigma_{0}}{n+2}\right)^{2} r^{2 n+4}-\Lambda r^{2}} .
$$

Equation (40) is not integrable, however, one can obtain the values of $t$ for different choices of $\frac{r^{*}}{\sqrt{\theta}}$ by numerical
Table 1 Values of $\frac{t}{\sqrt{\theta}}$ for different $\frac{r^{*}}{\sqrt{\theta}}$ (We have assumed $\frac{r_{h}}{\sqrt{\theta}}=10$,

\begin{tabular}{ll}
$\left.\frac{M}{\sqrt{\theta}}=1.8\right)$ & \\
\hline$\frac{r^{*}}{\sqrt{\theta}}$ & $\frac{t}{\sqrt{\theta}}$ \\
\hline 10 & 0 \\
10.5 & 2.3753 \\
11 & 3.5934 \\
11.5 & 4.4077 \\
12 & 5.01449 \\
12.5 & 5.4950 \\
13 & 5.8907 \\
\hline
\end{tabular}

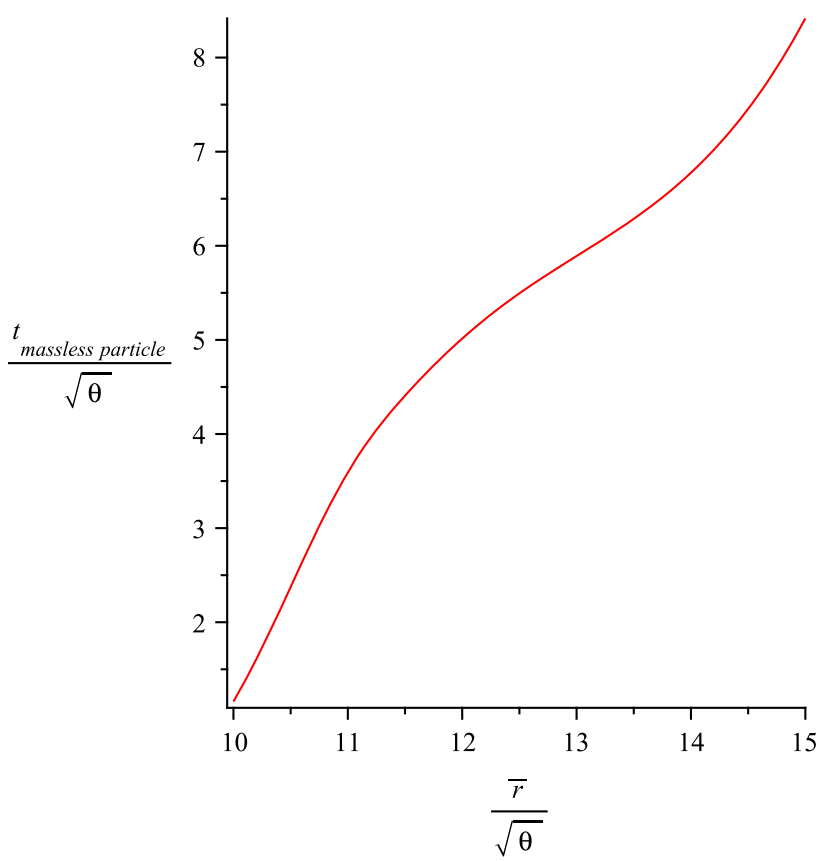

Fig. 11 Time $\frac{t}{\sqrt{\theta}}$ is plotted against $\frac{r}{\sqrt{\theta}}$

procedures. This is shown in Table 1 and a graphical representation of it is given in Fig. 11.

The effective potential of the system is obtained as

$$
\begin{aligned}
V_{\mathrm{eff}}= & -\frac{\Sigma^{2}}{2}+\frac{p^{2}}{2 r^{2}}\left[-M+2 M e^{-\frac{r^{2}}{4 \theta}}\right. \\
& \left.-\frac{1}{n+2}\left(\frac{4 \pi \sigma_{0}}{n+2}\right)^{2} r^{2 n+4}-\Lambda r^{2}\right]
\end{aligned}
$$

which for $p=0$ reduces to

$V_{\mathrm{eff}}=-\frac{\Sigma^{2}}{2}$

The nature of $V_{\text {eff }}$ for massless particles has been shown in Fig. 12. 
' $\frac{p}{\sqrt{\theta}}=0.05^{\prime} \cdot \frac{\Sigma}{\theta^{0.25}}=0.15^{\prime} \Lambda \sqrt{\theta}=-0.02^{\prime}{ }^{\prime} \sigma_{0} \theta^{0.75}=0.001^{\prime}{ }^{\prime} n=0$ '

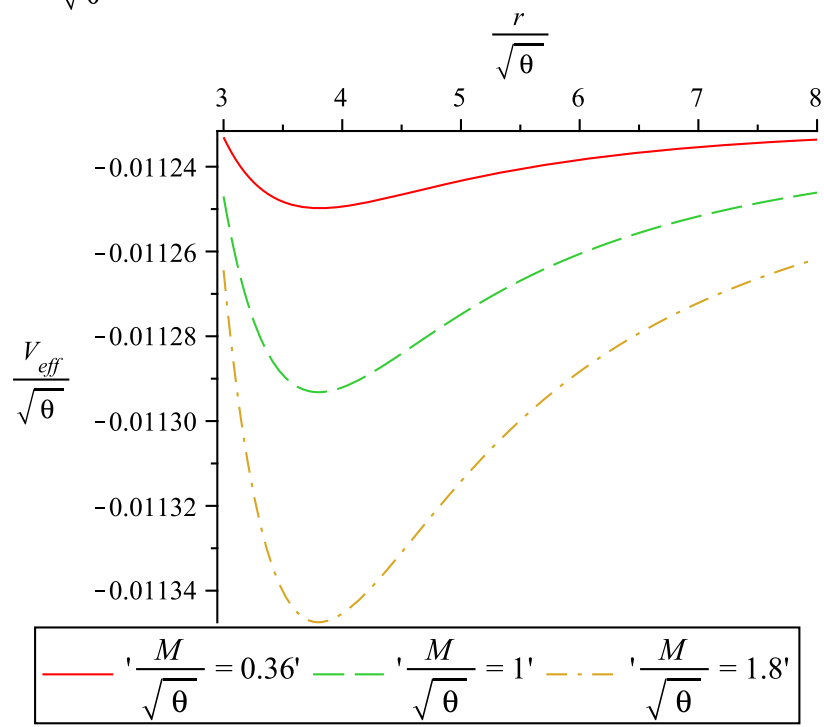

Fig. 12 Effective potential $\frac{V_{\text {eff }}}{\sqrt{\theta}}$ for massless particles. Here, $M=$ $0.36 \sqrt{\theta}$ (solid curve), $M=1 \sqrt{\theta}$ (dashed curve), and $M=1.8 \sqrt{\theta}$ (dot-dashed curve)

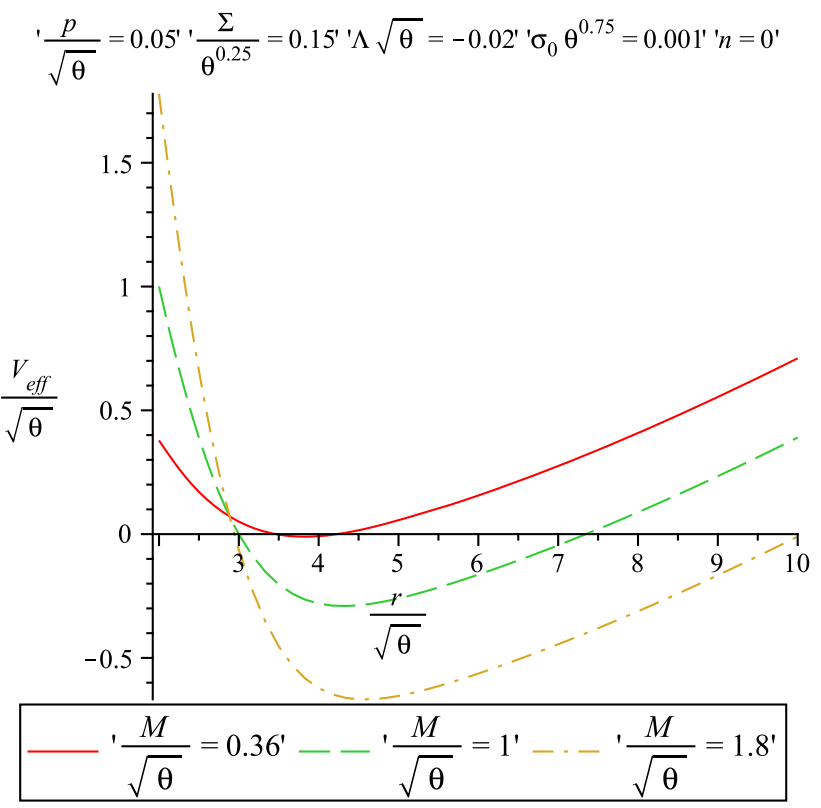

Fig. 13 Effective potential $\frac{V_{\text {eff }}}{\sqrt{\theta}}$ for massive particles. Here, $M=$ $0.36 \sqrt{\theta}$ (solid curve), $M=1 \sqrt{\theta}$ (dashed curve), and $M=1.8 \sqrt{\theta}$ (dot-dashed curve)

\subsubsection{Time-like geodesics}

For massive particles with $L=-1$ and $p=0$, we have

$\left(\frac{\mathrm{d} r}{\mathrm{~d} t}\right)^{2}=\left(\frac{f(r)}{\Sigma}\right)^{2}\left(\Sigma^{2}-f(r)\right)$ which in integral form can be written as

$\pm t=\Sigma \int \frac{\mathrm{d} r}{f(r) \sqrt{\Sigma^{2}-f(r)}}$.

For a time-like particle with $p \neq 0$, the effective potential $V_{\text {eff }}$ gets the form

$V_{\mathrm{eff}}=-\frac{1}{2} \Sigma^{2}+\frac{f(r)}{2}\left(1+\frac{p^{2}}{r^{2}}\right)$.

The nature of effective potential for massive particles is shown in Fig. 13. The shape of the effective potential indicates the possibility of stable circular orbits for massive particles around the black hole.

\section{Test particles}

Let us now analyze the motion of a test particle around noncommutative charged black hole using Hamilton-Jacobi (H-J) approach. Let $m$ and $e$ denote the mass and charge of the test particle. The H-J equation for the test particle has the standard form

$g^{i k}\left(\frac{\partial S}{\partial x^{i}}+e A_{i}\right)\left(\frac{\partial S}{\partial x^{k}}+e A_{k}\right)+m^{2}=0$,

where $g_{i k}$ is the background space and $S(t, r, \phi)$ is the Hamilton characteristic function. The gauge potential $A_{i}$ is given by

$A_{i}=\frac{Q \mathrm{~d} t}{r}$,

where $Q$ is the total charge of the black hole. For the background spacetime (1), the H-J equation gets the form

$$
-\frac{1}{f}\left(\frac{\partial S}{\partial t}+\frac{e Q}{r}\right)^{2}+f\left(\frac{\partial S}{\partial r}\right)^{2}+\frac{1}{r^{2}}\left(\frac{\partial S}{\partial \phi}\right)^{2}+m^{2}=0 .
$$

To solve the above partial differential equation, we use the method of separation of variables and choose the H-J function $S$ as

$S(t, r, \phi)=-E t+S_{1}(r)+p \phi$,

where $E$ is the energy and $p$ is the angular momentum of the particle. The radial velocity of the particle is then obtained as

$$
\frac{\mathrm{d} r}{\mathrm{~d} t}=f^{2}\left(E-\frac{e Q}{r^{2}}\right)^{-1} \sqrt{\frac{1}{f^{2}}\left(E-\frac{e Q}{r}\right)^{2}-\frac{m^{2}}{f}-\frac{p^{2}}{f r^{2}}} .
$$

Now, the turning points of the trajectory are determined from the requirement $\mathrm{d} r / \mathrm{d} t=0$, which yields

$$
\left(E-\frac{e Q}{r}\right)^{2}-m^{2} f-\frac{p^{2}}{r^{2}} f=0 .
$$




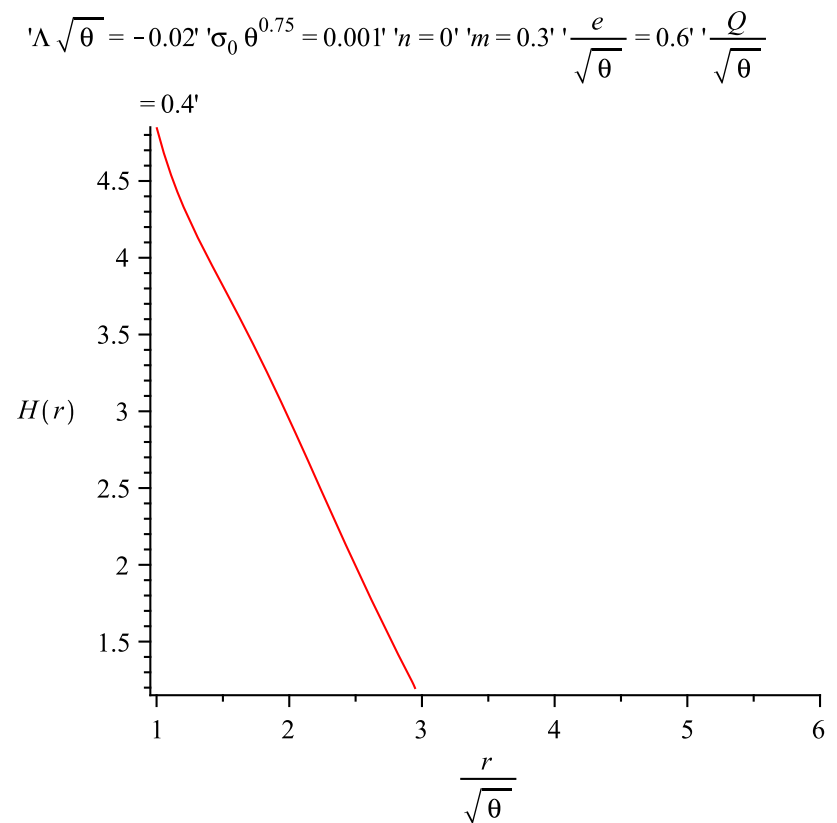

Fig. $14 H(r)$ is plotted against $\frac{r}{\sqrt{\theta}}$

Consequently, the potential curve can be obtained from the relation

$V(r) \equiv \frac{E}{m}=\frac{e Q}{m r}+\sqrt{f}\left(1+\frac{p^{2}}{m^{2} r^{2}}\right)^{1 / 2}$.

For a stationary system, $V(r)$ must have an extremal value, i.e., $\mathrm{d} V / \mathrm{d} r=0$, which implies

$\frac{e Q}{m r^{2}} \sqrt{f}\left(1+\frac{p^{2}}{m^{2} r^{2}}\right)^{1 / 2}=\frac{1}{2}\left(1+\frac{p^{2}}{m^{2} r^{2}}\right) f^{\prime}-f \frac{p^{2}}{m^{2} r^{3}}$.

\subsection{Test particle in static equilibrium}

In static equilibrium ( $p=0$ ), from Eq. (50), the value of $r$ at which potential curve will be an extremal can be obtained from the requirement

$$
\begin{aligned}
H(r) \equiv & \frac{e Q}{m r^{2}}\left[-M+2 M e^{-\frac{r^{2}}{4 \theta}}-\frac{1}{n+2}\left(\frac{4 \pi \sigma_{0}}{n+2}\right)^{2} r^{2 n+4}\right. \\
& \left.-\Lambda r^{2}\right]^{\frac{1}{2}}+\frac{M r}{2 \theta} e^{-\frac{r^{2}}{4 \theta}}+r^{2 n+3}\left(\frac{4 \pi \sigma_{0}}{n+2}\right)^{2}+r \Lambda=0 .
\end{aligned}
$$

A graphical representation of $H(r)$, as shown in Fig. 14, indicates that there does not exist any $r$ for which $H(r)=0$. This implies that the test particle cannot be trapped by the black hole. In other words, the black hole exerts no gravitation force on the test particle in a static equilibrium situation.

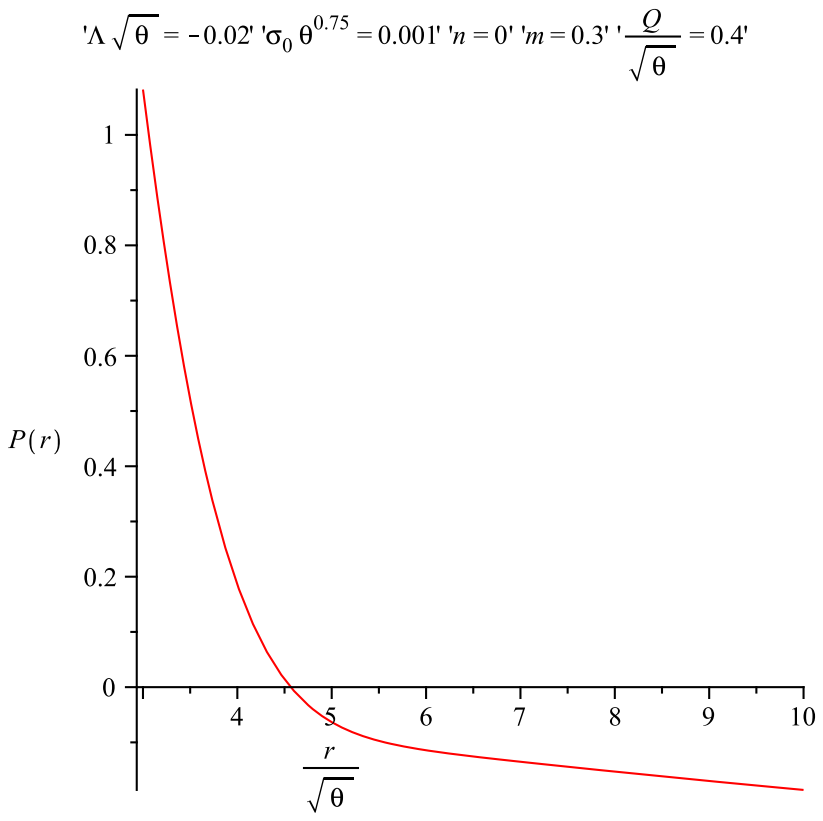

Fig. $15 P(r)$ plotted against $\frac{r}{\sqrt{\theta}}$

\subsection{Test particle in non-static equilibrium}

For a text particle in non-static equilibrium $(p \neq 0)$, we consider two possibilities: the uncharged and charged cases.

\subsubsection{Uncharged test particle $(e=0)$}

In this case, the value of $r$ at which potential curve will be an extremal can be obtained from the requirement

$$
\begin{aligned}
P(r) \equiv & \left(1+\frac{p^{2}}{r^{2} m^{2}}\right)\left[\frac{M r}{2 \theta} e^{-\frac{r^{2}}{4 \theta}}+r^{2 n+3}\left(\frac{4 \pi \sigma_{0}}{n+2}\right)^{2}+r \Lambda\right] \\
& -\frac{p^{2}}{m^{2} r^{3}}\left[-M+2 M e^{-\frac{r^{2}}{4 \theta}}-\frac{1}{n+2}\left(\frac{4 \pi \sigma_{0}}{n+2}\right)^{2} r^{2 n+4}\right. \\
& \left.-\Lambda r^{2}\right]=0
\end{aligned}
$$

In Fig. 15, we note that $P(r)=0$ at some finite value of $r$. Therefore, the particle can be trapped by the black hole. In other words, the black hole exerts gravitation force on the uncharged test particle in non-static equilibrium.

\subsubsection{Test particle with charge $(e \neq 0)$}

For a charged particle, the value of $r$ at which potential curve will be an extremal can be obtained from the requirement 


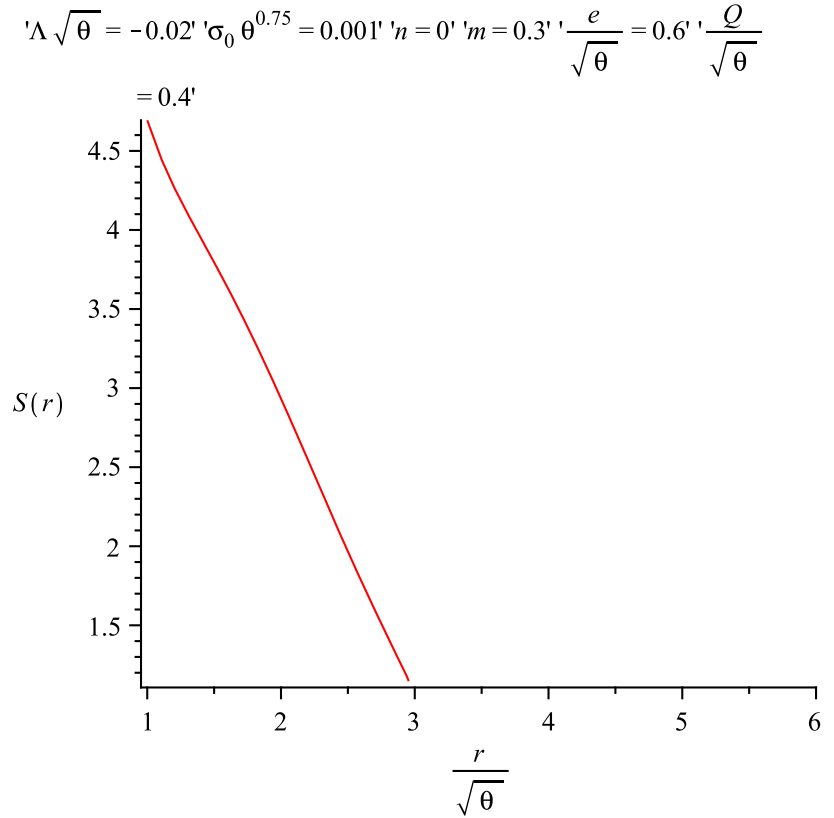

Fig. $16 S(r)$ is plotted against $\frac{r}{\sqrt{\theta}}$

$$
\begin{aligned}
S(r) \equiv \frac{e Q}{m r^{2}}\left(1+\frac{p^{2}}{m^{2} r^{2}}\right)^{\frac{1}{2}} \\
\times\left[-M+2 M e^{-\frac{r^{2}}{4 \theta}}-\frac{1}{n+2}\left(\frac{4 \pi \sigma_{0}}{n+2}\right)^{2} r^{2 n+4}-\Lambda r^{2}\right]^{\frac{1}{2}} \\
+\left(1+\frac{p^{2}}{r^{2} m^{2}}\right)\left[\frac{M r}{2 \theta} e^{-\frac{r^{2}}{4 \theta}}+r^{2 n+3}\left(\frac{4 \pi \sigma_{0}}{n+2}\right)^{2}+r \Lambda\right] \\
-\frac{p^{2}}{m^{2} r^{3}}\left[-M+2 M e^{-\frac{r^{2}}{4 \theta}}-\frac{1}{n+2}\left(\frac{4 \pi \sigma_{0}}{n+2}\right)^{2} r^{2 n+4}-\Lambda r^{2}\right]
\end{aligned}
$$$$
=0 \text {. }
$$

Figure 16 shows that $S(r) \neq 0$ for any finite value of $r$. This implies that the black hole exerts no gravitation force on the charged test particle in non-static equilibrium.

The results clearly show that a non-static charged test particle behaves differently from its neutral counterpart in the gravitational field of a charged black hole. The motion of a neutral particle is solely governed by the gravitational field of the black hole whereas the motion of a charged particle in the gravitational field of a charged black hole also experiences electromagnetic force. Therefore, while a neutral particle moves along the geodesic and gets trapped, a charged test particle in motion may not be trapped by the black hole.

\section{Discussions}

In this work, we have constructed a charged BTZ-like black hole solution by adopting the formalism of noncommutative geometry. The uncharged limit of this solution reported in
Ref. [19] can be regained simply by putting $\sigma=0$. The noncommutative effects on the physical quantities are found to be similar to the properties discussed in Ref. [19]. To investigate the impact of the electromagnetic field we have considered two cases $(\sigma=0$ and $\sigma \neq 0)$ and compared the results graphically. Our results show that the limiting mass $M_{0}$ below which no black hole can exist depends on its associated charge. In fact, the value of the limiting mass increases as charge is incorporated into the system. The location of the event horizon, Hawking temperature and heat capacity also do get modified in the presence of the electromagnetic field. Particle trajectories around the black hole are also affected by the inclusion charge. In particular, we note that the motion of a charged particle around a charged black hole is quite different from the motion of a neutral test particle. We hope that our results will contribute toward getting a better understanding of the effects of noncommutative geometry on the structure and properties of black holes. In addition, the results may be useful to understand how the presence of an electromagnetic field can change a number of astrophysical phenomena such as properties of accretion disks around charged black holes and Hawking radiation.

Acknowledgments RS and FR gratefully acknowledge support from the Inter-University Centre for Astronomy and Astrophysics (IUCAA), Pune, India, where a part of this work was carried out under its Visiting Research Associateship Programme. We are very grateful to an anonymous referee for his/her insightful comments that have led to significant improvements, particularly on the interpretational aspects.

Open Access This article is distributed under the terms of the Creative Commons Attribution License which permits any use, distribution, and reproduction in any medium, provided the original author(s) and the source are credited.

Funded by $\mathrm{SCOAP}^{3}$ / License Version CC BY 4.0.

\section{References}

1. E. Witten, Nucl. Phys. B 460, 335 (1996)

2. N. Seiberg, E. Witten, JHEP 032, 9909 (1999)

3. A. Smailagic, E. Spallucci, J. Phys. A: Math. Gen. 36, L517 (2003)

4. T.G. Rizzo, JHEP 09, 021 (2006)

5. P. Nicolini, A. Smailagic, P. Nicolini, Phys. Lett. B 670, 449 (2009)

6. Y.S. Myung, Y.W. Kim, Y.J. Park, J. High Energy Phys. 0702, 012 (2007)

7. M. Bañados, C. Teitelboim, J. Zanelli, Phys. Rev. Lett. 69, 1849 (1992)

8. K. Nozari, S.H. Mehdipour, Class. Quantum Gravity 25, 175015 (2008)

9. F. Rahaman, P.K.F. Kuhfittig, K. Chakraborty, A.A. Usmani, S. Ray, Gen. Relativ. Gravit. 44, 905 (2012)

10. F. Rahaman, P.K.F. Kuhfittig, S. Ray, S. Islam, Phys. Rev. D 86, 106010 (2012)

11. R. Banerjee, B.R. Majhi, S.K. Modak, Class. Quantum Gravity 26, 085010 (2009)

12. R. Tikekar, G.P. Singh, Gravit. Cosmol. 4, 294 (1998)

13. R. Sharma, S. Mukherjee, S.D. Maharaj, Gen. Relativ. Gravit. f33, 999 (2001) 
14. S. Thirukkanesh, S.D. Maharaj, Class. Quantum Gravity 25, 235001 (2008)

15. B.V. Ivanov, Phys. Rev. D 65, 104001 (2002)

16. F. Rahaman, A.A. Usmani, S. Ray, S. Islam, Phys. Lett. B 717, 1 (2012)

17. M. Cataldo, N. Cruz, S. del Campo, A. Garcia, Phys. Lett. B 484, 154 (2000)
18. A. Larrañaga, J.M. Tejeiro, Abraham Zelmanov J. 4, 28 (2011)

19. F. Rahaman, P.K.F. Kuhfittig, B.C. Bhui, M. Rahaman, S. Ray, U.F. Mondal, Phys. Rev. D 87, 084014 (2013)

20. J. Liang, B. Liu, Europhys. Lett. 100, 30001 (2012) 